\title{
Contribuições para a consolidação da New Public Governance: identificação das dimensões para sua análise
}

\author{
Breno Augusto Diniz Pereira ${ }^{1}$ \\ IVAN BeCK CKAgnazarofF ${ }^{2}$
}

\author{
${ }^{1}$ Universidade Federal de Santa Maria (UFSM) / Programa de Pós-Graduação em Gestão de Organizações Públicas e Programa \\ de Pós-Graduação em Administração Pública, Santa Maria - RS, Brasil \\ ${ }^{2}$ Universidade Federal de Minas Gerais (UFMG) / Departamento de Ciências Administrativas do Centro de Pós-Graduação e \\ PESQUISA EM AdMINISTRAÇÃO DA FACULDADE DE CIÊNCIAS ECONÔMICAS, BELO HORIZONTE - MG, BRASIL
}

\begin{abstract}
Resumo
Compreender a New Public Governance (NPG) como modelo de gestão vai ao encontro do atendimento às demandas atuais impostas pela sociedade às administrações públicas, em que há a necessidade de envolver os cidadãos no processo decisório das políticas públicas. Com isso, numa perspectiva macro, gerar informações sobre a evidenciação da NPG poderá contribuir para o desenvolvimento de estratégias que atendam às demandas que os cidadãos impõem à gestão, algo até então pouco efetivado pelos modelos existentes de administração pública. Dessa forma, este trabalho objetiva levantar dimensões para a análise da NPG, ainda carente na literatura. Conforme demonstrado, existem fundamentos para isso, entre eles: democracia deliberativa, coprodução e valor público. Por meio desses pressupostos, são analisadas dimensões sobre dois focos principais: estrutura e processos. Verificou-se que a NPG utiliza ferramentas dos modelos administrativos anteriores, mas busca sua ênfase dentro dos princípios democráticos e de legitimidade. Por fim, ressalta-se a necessidade de estudos teóricos e empíricos que possibilitem maior compreensão dessas ferramentas, permitindo uma administração pública mais inclusiva e com ações mais legítimas.
\end{abstract}

Palavras-chave: New Public Governance. Modelos de administração pública. Democracia. Legitimidade.

\section{Contributions to consolidate the new public governance: identifying dimensions of analysis}

\begin{abstract}
The current demands from society to public administration requires understanding new public governance (NPG) as a management model, involving citizens in the public policy decision-making processes. Therefore, from a macro-perspective, information on NPG may contribute to developing strategies to meet citizens' demands, which is not a usual practice in existing public administration models. This work identifies dimensions of NPG analysis, filling a gap in the literature. The foundations for NPG analysis are deliberative democracy, co-production, and public value, and the dimensions are identified based on structure and processes. The study found that NPG uses tools from previous administration models, focusing on democratic and legitimacy principles. Finally, the research emphasizes the need for theoretical and empirical studies to understand NPG tools better, enabling a more inclusive public administration with more legitimate actions.
\end{abstract}

Keywords: New public governance. Public administration models. Democracy. Legitimacy.

\section{Contribuciones a la consolidación de la Nueva Gobernanza Pública: identificación de las dimensiones para su análisis}

\section{Resumen}

La comprensión de la Nueva Gobernanza Pública (NGP) como modelo de gestión cumple con las demandas actuales impuestas por la sociedad a las administraciones públicas, donde es necesario involucrar a los ciudadanos en el proceso de toma de decisiones de las políticas públicas. Con esto, en una perspectiva macro, la generación de información sobre la divulgación de la NGP puede contribuir al desarrollo de estrategias para satisfacer las demandas que los ciudadanos imponen a la gestión, algo que hasta el momento no fue implementado de manera efectiva en los modelos de administración pública existentes. Así, este trabajo tiene como objetivo plantear dimensiones para el análisis de la NGP, que aún carece de literatura. Como se demostró, existen bases para el análisis de la NGP que son: democracia deliberativa, coproducción y valor público. Mediante estos supuestos, se analizan las dimensiones desde dos enfoques principales: estructura y procesos. Se verificó que la NGP utiliza herramientas de modelos administrativos anteriores, pero busca su énfasis dentro de los principios democráticos y de legitimidad. Finalmente, también se enfatiza la necesidad de estudios teóricos y empíricos que permitan una mayor comprensión de estas herramientas, permitiendo así una administración pública más inclusiva con acciones más legítimas.

Palabras clave: Nueva Gobernanza Pública. Modelos de administración pública. Democracia. Legitimidad. 


\section{INTRODUÇÃO}

Governança se tornou a palavra da moda na área de administração pública. No meio acadêmico ou na prática, ela é a "ferramenta" para que os gestores possam implementar as políticas públicas com maior efetividade (Hufty, 2010). O objetivo deste trabalho é apresentar uma proposta para análise da governança considerando a evolução dos paradigmas de administração pública frente a uma democracia mais cidadã.

Compreende-se que a governança pública se inspira em teorias democráticas, preocupadas com maior participação nos processos deliberativos nos espaços públicos e com a necessidade de eficiência e legitimidade democrática dos governos (Lynn \& Malinowska, 2018). Dessa forma, a governança pública, ou New Public Governance (NPG), como modelo, e não como ferramenta de gestão aplicada na administração burocrática e na New Public Management (NPM), pode contribuir para a consolidação de uma democracia mais inclusiva, que aponte a construção de cidadãos políticos e a expansão da esfera pública.

Para Kissler e Heidemann (2006) e Paula (2005), a governança pública está associada a uma mudança nas dimensões sociopolítica, econômico-financeira e institucional-administrativa, pois aponta para a consolidação dos processos de coprodução e cogestão do social na gestão pública, enfatizando a participação ativa, a ação conjunta e a inclusão dos cidadãos na comunidade política, bem como reconhecendo os excluídos como cidadãos e gerando espaços públicos de participação e controle social. Governança pública expressa uma vontade política de implementar um projeto democrático que possibilite maior articulação entre Estado e sociedade civil, assim como vontade política de construção de espaços públicos - que implementem de forma efetiva a participação - e de ampliação da política, na medida em que considera a importância da sociedade civil na decisão fruto de debate e deliberação ampliada - e na formulação de políticas públicas (Dagnino, Olivera \& Panfichi, 2006).

Neste trabalho, governança pública é entendida como referente à relação entre governantes e governados, associada à coprodução do desenvolvimento, mas principalmente à construção de uma democracia mais inclusiva que possibilite ao cidadão construir, coordenar e avaliar as políticas públicas. Refere-se, portanto, à dimensão governamental, ou seja, a um tipo de arranjo institucional governamental que, ao articular as dimensões econômico-financeira, institucional-administrativa e sociopolítica, estabelecendo parcerias com sociedade civil e mercado por meio de processos deliberativos, busca soluções inovadoras para os problemas sociais.

\section{BREVE CONTEXTUALIZAÇÃO DO PENSAMENTO SOBRE GOVERNANÇA PÚBLICA}

Na segunda metade do século XX, frente ao intenso ritmo do sistema capitalista que assumiu as relações sociais e econômicas, o movimento da administração pública burocrática se mostrou limitado pela sua obediência cega às normas, gerando rigidez na atuação. Isso ocasionou movimentos de maior flexibilidade do Estado e estimulou a mudança no processo para o foco nos resultados, dando os primeiros sinais da reforma que orientaria a transição do movimento de administração burocrática para a administração pública gerencial, ou New Public Management (NPM).

A NPM representa um conjunto de premissas e valores sobre como organizações públicas devem ser constituídas, organizadas, gerenciadas e, semelhante aos negócios privados, funcionar (Diefenbach, 2009). Ao focar na eficiência, no controle e na gestão por resultados, a NPM adota uma perspectiva tecnocrática, tendo como base uma independência necessária entre a política e a gestão pública, revisitando a dicotomia política-administração pública (Wiesel \& Modell, 2014).

De acordo com R. B. Denhardt e J. V. Denhardt (2003) e Radnor, Osborne e Glennon (2016), no entanto, a NPM enfrenta uma série de problemas. Primeiro, a sociedade se transformou profundamente desde o início da NPM, na década de 1980; tornou-se mais fragmentada, sendo cada vez mais difícil, se não impossível, organizações públicas responderem isoladamente às necessidades sociais. Dessa forma, o Estado não atua mais como um único agente na entrega de serviços públicos; ao contrário, necessita da colaboração de diversos atores para a prestação dos serviços. Por consequência, não pode mais agir como se sua eficiência e eficácia estivessem em suas próprias mãos. Com grande frequência, as reformas ao estilo NPM têm se concentrado só na eficiência interna, em vez de focadas na eficácia em atender às necessidades expressas de usuários de serviços e cidadãos (Radnor et al., 2016). 
Em segundo lugar, na NPM a tarefa de prestação de serviços públicos foi conceituada como algo a ser realizado por profissionais de serviço público, num local onde o usuário do serviço é amplamente passivo no processo, ou seja, um "cliente". R. B. Denhardt e J. V. Denhardt (2003) salientam que "as organizações públicas, já que estão envolvidas na expressão dos valores societários, devem conceder a seus membros uma parte do poder de tomar decisões". Para Carneiro e Menicucci (2011), a sociedade requer um Estado ativo, necessitando de ferramentas e formas de organização que dialoguem com a sociedade e garantam, de forma efetiva, a resolução de seus problemas.

Por fim, a NPM não foi capaz de responder aos desafios das práticas orientadas pelo conhecimento e pela prestação de serviços na economia digital. Isso não está apenas transformando a natureza de relações entre organizações públicas e usuários de serviços, mas também questionando a valorização do público como indicador da eficácia do serviço público, em vez de medidas internas de eficiência do serviço público e processos de governança necessários para negociar um acordo sobre essa eficácia (Brown \& Osborne, 2013).

Aliado a isso, uma série de novos desafios transversais para os Estados - como a expansão de medidas preventivas de cuidados de saúde e a melhoria da segurança pública e da empregabilidade - veio à tona (Klijn \& Koppenjan, 2004). Problemas de migração de povos, por exemplo, com frequência não podem ser solucionados pelas políticas de um único país, pois dependem da articulação de um conjunto de nações, além de um aparato de órgãos públicos federais, estaduais e municipais, como a parceria com organismos internacionais como ONU e diversas organizações sociais locais trabalhando em conjunto.

Esses problemas demonstraram a incapacidade do Estado na aplicação da NPM e do modelo burocrático no que diz respeito a resolver os problemas complexos e não solucionáveis pelas estruturas hierárquicas da administração pública. Isso fortaleceu o apelo à coordenação horizontal por meio de interação entre atores públicos e privados.

Dessa forma, percebe-que a administração pública necessita de novas iniciativas ou modelos para a ação estatal, retirando do Estado a exclusividade na atenção às demandas da sociedade. $O$ avanço dessas abordagens vem sendo tratado pela vertente da governança pública, que, como uma de suas principais características, considera um Estado plural, no qual vários atores interdependentes contribuem para a prestação de serviços públicos, e também pluralista, em que vários processos e atores influenciam o sistema de tomada de decisões das políticas públicas.

Para Osborne (2006), a gestão pública contempla cada vez mais uma articulação interorganizacional, envolvendo um conjunto de atores que participam da execução das políticas públicas e de identificação e concepção, o que remete à necessidade de uma visão sistêmica e processual, em substituição a uma visão pontual e limitada dos problemas sociais. A NPG muda as relações institucionais entre setor público e sociedade, ampliando as redes entre organizações dentro e fora do governo para fornecer serviços públicos. A emergência de relações não hierárquicas inibe os sistemas de comandos formais, demandando a construção de vínculos baseados na confiança e em contratos relacionais não formais, segundo expectativas e possibilidades das partes. O Estado se torna, enfim, um organismo com ação plural, exigindo mudanças na sua forma de ação e na construção de metodologias a serem utilizadas para compreender tanto seu papel nesse novo modelo quanto esse novo sistema de coordenação (Head \& Alford, 2015).

Peters e Pierre (1998) analisam que, enquanto a NPM está relacionada a resultados, a NPG trata mais de processos e estrutura. Ademais, afirmam que a NPG, como qualquer outro modelo, é influenciada pelo ambiente político e cultural, podendo se configurar de distintas estruturas segundo os contextos nacionais. Já a NPM pretende certa neutralidade em relação ao contexto em que seria aplicada, um conjunto de princípios e proposições que poderiam ser aplicados em qualquer realidade, sem problemas de adaptação. Enquanto a NPM se volta para processos intraorganizacionais, a NPG se volta para ações interorganizacionais.

Além disso, a NPG considera que as formas participativas e deliberativas de democracia podem colaborar para o enfrentamento dos problemas complexos da administração pública, possibilitando a ampliação das capacidades do Estado (Carneiro \& Menicucci, 2011). Para isso, as organizações públicas do Estado devem ser permeadas pelo objetivo da responsividade e do valor público tanto quanto devem corresponder aos anseios públicos, ou seja, acima dos interesses das classes e dos grupos sociais. Essa compreensão faz com que os estudos sobre NPG recaiam sobre as questões associadas à gestão da mudança em sistemas complexos, englobando, assim, o contexto social, político e ético mais amplo que envolve a administração pública. 


\section{Dimensões de análise da NPG}

O modelo da NPG busca que o Estado trabalhe em prol da conciliação de interesses dispersos voltados a uma administração pública mais eficiente, responsiva e democrática. Conforme Osborne (2010) e Wiesel e Modell (2014), os princípios do modelo burocrático e da NPM continuam existindo e se configuram como ferramentas importantes para compreender o processo de construção da NPG.

Para Bingham, Nabatchi e O'Leary (2005) e Sorrentino, Sicilia e Howlett (2018), a NPG é um modelo de administração pública capaz de conciliar interesses de uma gestão eficiente e eficaz, com maior inserção das bases democráticas para a construção, a gestão e a implementação das políticas públicas. Nas sociedades avançadas, a inclusão nos processos de governança de uma pluralidade de atores, além do setor público, é considerada uma forma prática de abordar problemas cada vez mais complexos (Sorrentino, Marco \& Rossignoli, 2016). Assim, o engajamento dos cidadãos no ciclo de serviço público revigora a realização de objetivos públicos e é visto como uma opção eficaz para a moderna administração pública. Em outras palavras, a NPG vem expor as bases fundamentais para a construção do espaço público, introduzindo novas estruturas, novos processos de gestão e uma democracia mais inclusiva para as deliberações e as construções das políticas públicas.

Dessa forma, há de ressaltar os fundamentos básicos que compõem o modelo da NPG, ou seja, suas dimensões essenciais que a distinguem dos outros modelos de administração pública. Essas dimensões, conforme exposto ao longo do trabalho, podem favorecer uma maior cidadania e participação nas decisões. Assim, um modo de abordagem da NPG demanda a análise dos seguintes construtos: democracia deliberativa, valor público das decisões e coprodução das decisões.

\section{Democracia deliberativa}

Habermas (1995), ao elaborar o conceito de democracia deliberativa, está preocupado com o modo com o qual os cidadãos fundamentam racionalmente as regras do jogo democrático. Buscam-se criar condições para legitimar a democracia onde a teoria do discurso propõe um "procedimento ideal para a deliberação e a tomada de decisão". Para Faria (2000), tal procedimento democrático, ao conjugar considerações pragmáticas, compromissos, discursos de autocompreensão e de justiça, fundamenta o pressuposto de que resultados racionais e justos serão obtidos por meio de um fluxo de informação relevante cujo emprego não deve ser obstruído.

A operacionalização desse procedimento ideal de deliberação e tomada de decisão, ou seja, das políticas deliberativas, depende, segundo a teoria do discurso, da institucionalização dos procedimentos e das condições de comunicação, bem como da inter-relação de processos deliberativos institucionalizados com as opiniões públicas informalmente constituídas.

Bohman (1996, p. 183) salienta, nesse contexto, a questão da legitimidade. Para ele, a legitimidade de "uma lei ao resultado de um processo participativo justo e aberto a todos os cidadãos e que, como tal, inclui todas as razões publicamente acessíveis dessas pessoas". O autor não pressupõe um acordo unânime entre cidadãos ou legisladores sobre todas as leis, objetivos ou decisões, e sim uma cooperação contínua a despeito das diferenças de posições próprias de uma sociedade pluralista. A constituição do consenso, nessa versão, dependerá da participação no processo público e legislativo, independentemente do desacordo em relação a qualquer decisão particular alcançada de maneira deliberativa. Ao participar de um processo deliberativo justo, inclusivo e cooperativo, os cidadãos chegarão racional e publicamente a uma ação legítima (Faria, 2000).

Para compreender a legitimidade democrática, portanto, precisa-se adicionar uma dimensão moral: aqueles que concedem a legitimidade devem fazê-lo porque acreditam que é moralmente correto. Para Dryzek (2010) e Sant'Anna, Alcântara, Pereira, Cappelle e Tonelli (2019), também é necessário adicionar uma dimensão da liberdade: quem concede legitimidade deve fazê-lo livremente. Precisa-se, então, adicionar uma dimensão de competência: aqueles que concedem legitimidade devem fazê-lo com plena consciência do que estão sendo solicitados a aceitar. Assim, legitimidade não significa apenas aceitação; refere-se também à retidão moral, à liberdade e à competência no processo de aceitação.

\section{Valor público}

A NPG vê o valor público emergindo de diálogos e deliberações amplamente inclusivos. No entender de Bozeman (2007), os valores públicos de uma sociedade são aqueles que fornecem consenso normativo sobre: "(1) os direitos, benefícios e prerrogativas aos quais os cidadãos devem (e não devem) ter; (2) as obrigações dos cidadãos para com a sociedade, o estado e entre si; e (3) os princípios nos quais os governos e as políticas devem se basear." 
Para Alford e Hughes (2008) e Jørgensen e Bozeman (2007), o valor público pode ser produzido por organizações governamentais, empresas privadas, organizações sem fins lucrativos ou voluntárias, usuários de serviços ou várias outras entidades. Não é quem o produz que torna público o valor. Pelo contrário, é uma questão de quem o consome. Como Moore (1995) explica, o valor público é o valor "consumido" coletivamente pelos cidadãos, e não individualmente pelos "clientes". Isso envolve mais do que bens públicos conforme definido pelos economistas. No mínimo, inclui também soluções para "falhas de mercado" de vários tipos, isto é, para situações em que os mecanismos de mercado não maximizam o bem-estar individual dos cidadãos, como externalidades negativas, monopólios naturais ou informações imperfeitas. Ao mesmo tempo, os cidadãos também valorizam os arranjos institucionais que permitem que os mercados operem e as ordens sociais funcionem, como o Estado de direito, a manutenção da ordem e os mecanismos para a proteção dos direitos de propriedade e a aplicação da lei.

Em primeiro lugar, uma razão pela qual os cidadãos valorizam os arranjos é porque eles os beneficiam pessoalmente. Mas, em muitos casos, também as valorizam, e por motivos que vão além do interesse individual. Eles têm objetivos ou aspirações para a sociedade como um todo, fundamentados em compromissos ou propósitos sociais ou normativos (Moore, 1995), como justiça, orgulho nacional, cuidado com o meio ambiente ou preocupação com desprovidos e vulneráveis.

Em segundo lugar, o que Bozeman (2007) chama de "falha de mercado" ocorre quando nem o mercado nem o setor público fornecem bens e serviços para atingir valores públicos necessários. Os valores públicos para Bozeman (2007) podem, portanto, ser mensuráveis, embora haja divergências sobre como eles devem ser conceituados e medidos (Bryson, Crosby \& Bloomberg, 2014). Uma implicação é que analistas, cidadãos e formuladores de políticas devem se concentrar no que são valores públicos e nas maneiras pelas quais instituições e processos são necessários para estabelecer acordos e atingir valores públicos na prática (Davis \& West, 2009; Kalambokidis, 2014).

Moore (1995) argumenta que os gestores públicos devem usar o que ele chama de triângulo estratégico. A estratégia deve ser (1) destinada a alcançar algo substancialmente valioso (isto é, deve constituir valor público), (2) legítimo e politicamente sustentável, (3) operacional e administrativamente viável. Moore "iguala sucesso gerencial no setor público ao iniciar e remodelar organizações do setor público de maneira a aumentar seu valor para o público no curto e no longo prazo", o que requer uma "imaginação inquieta e que busca valor".

Para Moore (1995), assim como para Bozeman (2007), o valor público geralmente se refere a estados objetivos do mundo que podem ser medidos. Algo que está sendo avaliado pode ser considerado um valor inerente ou um meio para outra coisa. Sintetizando, os autores resumem o conjunto de pressupostos em que a eficiência organizacional pública, a responsabilidade, a justiça e a justiça no contexto da governança democrática são valores primordiais.

\section{Coprodução}

No entender de Amamoto, Pereira e Alcântara (2020), coprodução é um conjunto de atividades que conta com a participação de agente públicos e de cidadãos, que constroem conjuntamente políticas e ações públicas, buscando a melhoria na prestação de serviços. Nabatchi, Sancino e Sicilia (2017) veem coprodução como necessária numa época de austeridade, ao passo que outros a veem como uma forma de redefinir os papéis de governo, usuários da sociedade civil, cidadãos, e de serviço (Nabatchi, Steen, Sicilia \& Brand, 2016). Ainda há os que argumentam que ele cria valor público: tem valor instrumental para as organizações em termos de melhoria de eficiência, eficácia e inovação (Osborne, Radnor \& Nasi, 2013); valor instrumental para cidadãos em termos de satisfação e capacitação (Sancino, 2016); capital social (Jakobsen, 2013); e prestação de contas (Ostrom, 1996).

Nabatchi et al. (2017) afirmam que a coprodução envolve dois tipos de participantes: (1) agentes estatais que são agentes (diretos ou indiretos) do governo possuidores de uma capacidade profissional (ou seja, os "produtores regulares") e (2) atores que são membros do serviço público voluntariamente como cidadãos, clientes e/ou consumidores (ou seja, os "cidadãos produtores"). Assim, na coprodução, os agentes estatais sempre estarão presentes, de maneira indireta ou direta. Já os cidadãos produtores podem participar de forma individual ou coletiva.

De acordo com Osborne, Radnor e Strokosch (2016), a importância da coprodução deriva não só do seu papel na contribuição para o impacto e a eficácia dos serviços públicos, mas também do seu papel de bem-estar que resulta dessa atividade e do seu potencial para facilitar a evolução da capacidade individual e coletiva em responder de forma independente a necessidades 
sociais no futuro (cocriação). Essa cocriação de valor é fundamental para os serviços públicos que são capazes de verificar uma contribuição do cidadão de modo mais amplo, viável e eficaz para a sociedade, agora e no futuro. Está no coração do desenvolvimento de serviços públicos sustentáveis no século XXI.

A seguir, é apresentado o Quadro 1, o qual expõe as dimensões para a compreensão do processo da NPG. Não se busca aqui esgotar o tema com a definição dos referidos construtos, e sim possibilitar uma visão mais abrangente sobre os aspectos da NPG que podem ser analisados.

\section{Quadro 1}

Dimensões de análise da Nova Governança Pública (NPG)

\begin{tabular}{|c|c|c|c|}
\hline Dimensões & Característica & Categorias De Análise & Autores \\
\hline \multirow{3}{*}{$\begin{array}{c}\text { ELEMENTOS } \\
\text { FUNDAMENTAIS } \\
\text { BÁSICOS }\end{array}$} & \multirow{3}{*}{$\begin{array}{l}\text { Caracterizam as principais } \\
\text { preocupações da governança, } \\
\text { servindo como valores ou } \\
\text { princípios norteadores esperados } \\
\text { da nova governança pública. }\end{array}$} & $\begin{array}{l}\text { Democracia } \\
\text { Deliberativa }\end{array}$ & $\begin{array}{c}\text { Habermas (1995); } \\
\text { Dryzek (2010). }\end{array}$ \\
\hline & & Valor Público & $\begin{array}{c}\text { Bozeman (2007), } \\
\text { Moore (1995). }\end{array}$ \\
\hline & & Coprodução & $\begin{array}{c}\text { Ostrom (1972); } \\
\text { Nabatchi et al. (2017); } \\
\text { Alford (2009); } \\
\text { Osborne et al. (2016). }\end{array}$ \\
\hline \multirow{3}{*}{ ESTRUTURA } & \multirow{3}{*}{$\begin{array}{l}\text { Consiste em assegurar que as } \\
\text { organizações públicas, ou os } \\
\text { diversos sistemas institucionais } \\
\text { e gerenciais que integram a } \\
\text { administração pública, atuem } \\
\text { adequadamente, de forma } \\
\text { conjunta e interativa, para obter } \\
\text { os resultados desejados. }\end{array}$} & $\begin{array}{l}\text { Deslocamento } \\
\text { do Poder }\end{array}$ & $\begin{array}{l}\text { Pierre e Peters (2010); } \\
\text { Jessop (1993). }\end{array}$ \\
\hline & & Redes & $\begin{array}{c}\text { Rhodes (1997); } \\
\text { Provan e Kenis (2008). }\end{array}$ \\
\hline & & Coordenação & $\begin{array}{c}\text { Bass (1985); } \\
\text { Bryson e Crosby (1992). }\end{array}$ \\
\hline \multirow[b]{2}{*}{ PROCESSOS } & \multirow{2}{*}{$\begin{array}{l}\text { Conjunto de medidas tomadas } \\
\text { para avaliar, monitorar e } \\
\text { comunicar os resultados das } \\
\text { políticas públicas criadas e } \\
\text { implementadas. Realiza-se um } \\
\text { juízo sobre seu valor ou mérito } \\
\text { considerando a relevância dos } \\
\text { objetivos e estratégias adotadas. }\end{array}$} & Eficiência e Eficácia & $\begin{array}{c}\text { Ridley e Simon (1937); } \\
\text { Christensen e Lægreid (2011). }\end{array}$ \\
\hline & & Accountability & $\begin{array}{c}\text { Skelcher e Smith (2015); } \\
\text { R. B. Denhardt e } \\
\text { J. V. Denhardt (2003); } \\
\text { Christensen e Lægreid (2007). }\end{array}$ \\
\hline
\end{tabular}

Fonte: Elaborado pelos autores.

Após a compreensão dos fundamentos básicos que sustentam a NPG, busca-se sua estruturação propriamente dita. Conforme Dias (2012), a NPG pode ser estruturada sob duas perspectivas principais: numa óptica estruturalista, já que essas interações se dão em meio a diferentes desenhos de estruturas e realidades, e como um processo dinâmico de cooperação, dada a presença considerável da interatividade. Essas perspectivas vão ao encontro da visão de Osborne (2010) e Wiesel e Modell (2014), que sustentam que a NPG não rompe os modelos de administração pública anteriores, e sim os aperfeiçoam, com a introdução dos fundamentos básicos para sua análise. Dessa forma, trata-se de uma nova visão sobre os conceitos atribuídos pela administração pública burocrática e pela NPM, com características de democracia, coprodução e valor público.

Para Jessop (1993), do ponto de vista da estrutura, podem-se analisar os seguintes pontos: deslocamento do poder, redes e coordenação. Com relação ao deslocamento do poder, identificam-se três direções em que essa realocação pode ocorrer: a) um deslocamento do poder do sistema político central para sistemas regionais e locais (downward); b) um deslocamento do poder para cima, no sentido de organizações transnacionais - como a União Europeia e a Organização Mundial do Comércio (OMC) - (upward); e c) um deslocamento do controle para instituições externas com devido poder discricionário que atuam como extensão da competência do Estado (outward) (Pierre \& Peters, 2010).

Se os movimentos downward, upward e outward são vistos por alguns como perda de poder por parte do Estado, Pierre e Peters (2010) advertem que isso só acontece porque o próprio Estado concorda, dada a complexidade dos problemas atuais. 
No entendimento deles, esse poder é delegado de maneira consciente pelo Estado, por isso não corresponderia à diminuição da importância de seu papel nem de sua capacidade como garantidor do desenvolvimento.

Na NPG, segundo Pierre e Peters (2010), ainda que o Estado "autorize" o deslocamento do poder e do controle, mantém uma estrutura institucional desenhada para garantir que a formulação e a implementação das políticas públicas sejam projetadas para o território de maneira inteira. Em outras palavras, ele estaria delegando o poder no sentido de que cada local ou região se desenvolva, mas nem por isso descola da ideia de que o local ou a região são parte de um todo e que esse todo é de competência e responsabilidade do governo central (Dias, 2012).

A formação de redes é outra das características mais marcantes para a NPG. As redes vêm se tornando cada vez mais relevantes, já que são uma forma de administrar políticas e projetos que envolvem problemas complexos, recursos escassos, diversos atores e interação de agentes públicos e privados. Embora as redes sejam conceituadas de várias maneiras, a maioria das definições tem certas características comuns: são caracterizadas por problemas políticos complexos que não podem ser resolvido apenas por um ator, exigindo ações coletivas de vários atores (Koppenjan \& Klijn, 2004); têm interdependências relativamente altas entre os atores, porque recursos necessários para resolver problemas pertencem a diferentes atores (Hanf \& Scharpf, 1978); essas interdependências causam um alto grau de complexidade estratégica e um curso imprevisível de interações ( $\varnothing$ rensen \& Torfing, 2007), como ações de um ator afetam interesses e estratégias de outros atores; as interações de rede mostram alguma durabilidade ao longo do tempo (Agranoff \& McGuire, 2003).

Rhodes (1997) descreve como as redes podem ser caracterizadas com graus de estabilidade, em relação à amplitude da rede e a uma maior ou menor horizontalização na articulação. Ele adapta uma estrutura de Marsh e Rhodes (1992) que distingue o tamanho da rede com base no número de membros, os tipos de interesses que os membros trazem para a rede, a frequência de suas interações, a continuidade e existência de um consenso entre eles e a distribuição de recursos dentro da rede e entre os atores participantes.

Já com relação à coordenação das redes, Souza e Hollanda (2019) ressaltam que ela raramente é efetivada de forma espontânea. Quando informal, sujeita à baixa efetividade e durabilidade, de forma que requer uma coordenação efetiva para que seja estruturada e orientada para os objetivos do Estado (Provan \& Kenis, 2008). Assim, os "governos coordenados" são essenciais por passar a enfatizar o compartilhamento de informações e a coordenação de esforços entre os envolvidos na resolução dos problemas.

A estrutura das redes na NPG leva ao desenvolvimento de um sistema multicamada de soberania compartilhada. Esse desenvolvimento destaca deficiências nas teorias tradicionais da democracia. De acordo com Koliba, Meek e Zia (2010), faz-se necessário promover a democracia num sistema político baseado em soberania compartilhada, determinando como a competência para tomada de decisões deve ser dividida não dentro de uma entidade política predeterminada, mas entre unidades políticas relativamente autônomas de governança social. Em outras palavras, precisam-se construir diretrizes direcionando os esforços para construir dinamicamente "as pessoas" em processos concretos de tomada de decisão.

Vários autores (Kelly \& Muers, 2002; Moore, 1995; Stoker, 2003) argumentaram que, para a efetivação da NPG, os agentes públicos devem procurar atuar com o que Ramadass, Sambasivan e Xavier (2018) denominaram de líderes transformacionais. Deve-se enfatizar que os líderes transformacionais são essencialmente exercidos por meio de redes de governança. Então, para criar valor público, os agentes públicos devem assumir a responsabilidade de coordenar redes em sua busca.

Para Bass (1985), as características de um líder transformacional relevantes para colaboração do setor público são: foco em prioridades e objetivos importantes; criar um clima ético, visto que o objetivo da colaboração no setor público é resolver problemas sociais; promover uma forte colaboração; garantir governança adequada; incentivar os parceiros a trabalhar para o bem comum e alcançar os resultados desejados.

A literatura em desenvolvimento sobre liderança no campo da administração pública tende a vê-los como principalmente baseados em interesses individuais ou coletivos. Nessa perspectiva, o papel da liderança do agente público visa à coordenação dos atores envolvidos no processo de governança, mobilizando e reunindo seus dispersos recursos em face de "estruturas de dependência de recursos" (Rhodes, 1988). Uma complementaridade de interesses pode surgir assim de uma estrutura relativamente equilibrada de dependências de recursos, podendo gerar contribuições significativas de recursos no contexto de uma questão política em andamento ou séries de questões interconectadas. 
À medida que as negociações se consolidam, outros fatores, como confiança e cooperação, podem se desenvolver, já que os atores perceberão que a deserção em qualquer área pode levar ao desenrolar da cooperação em outras áreas. Os líderes disponibilizam um verdadeiro senso de propósito para os membros, bem como a colaboração que energiza esses membros para alcançar objetivos (Ramadass et al., 2018). Por sua vez, os membros das redes fornecem maior valor para a governança e para os outros atores, buscando atingir as metas da sua colaboração (Konorti, 2012).

Outro fator para obter êxito na elaboração, na aplicação e no monitoramento da governança e que contribui para as políticas públicas eficazes é a análise dos processos de ponta a ponta (Hernaus, Vuksic \& Štemberger, 2016). Silva (2020) ressaltou que não se pode analisar a eficiência sem associá-la com a efetividade, pois otimizar recursos públicos sem oferecer bons serviços ou sem resolver os problemas sociais é incompatível com os valores definidos numa democracia. Todavia, as ferramentas utilizadas para analisar os processos são ressaltadas na perspectiva da NPM, como avaliação de eficiência e eficácia e accountability (Sant'Anna et al., 2019; Silva, 2020). No entanto, essas ferramentas/construtos são ampliadas na visão da NPG, pois, além de incluir resultados econômicos, ou seja, apenas uma lógica de análise, visam à conciliação com os resultados sociais e democráticos. Para a NPG, pode-se ter uma melhor utilização de recursos tanto em termos de eficácia quanto de efetividade. Considerando o modo pelo qual as deliberações e as decisões foram tomadas, garantindo a legitimidade do processo, considerando a complementariedade de recursos entre os atores envolvidos e a prestação de contas, é possível que políticas públicas sejam mais eficazes e efetivas.

Um dos grandes avanços que a NPG traz em relação ao modelo burocrático e à NPM é o olhar da sociedade para a análise dos resultados da administração pública. Enquanto o modelo burocrático focou quase exclusivamente na eficiência, deixando de se preocupar com os resultados (eficácia), a NPM foi um movimento em direção aos resultados. Só que esses resultados, como retratado na literatura, estão mais voltados aos resultados da gestão em si, como se, ao ser eficaz economicamente, seriam eficazes democraticamente (Christensen \& Lægreid, 2011; Ter Bogt, 2008). Além disso, estudos mostram que a NPM incentivou a proliferação de sobreposições entre diferentes entidades públicas, o que levou ao aumento de custos e à redução de eficiência no setor público (Diefenbach, 2009; Rhodes, 1996).

Já para a NPG, o pressuposto da avaliação dos resultados é diferente. A avaliação dos resultados nessa perspectiva deve ser vista como um processo orientado para julgar a valoração e os méritos de iniciativas políticas governamentais, cujos resultados são capazes de expressar tanto os conflitos quanto as conciliações sociais desenvolvidas em diferentes contextos e instituições, sendo expresso pelo valor agregado a sociedade. Na NPG está contida a ideia do valor público, cuja preocupação vai além de ser economicamente eficiente. Buscam-se fins socialmente desejados por meio de um incentivo à participação em ambientes democráticos e deliberativos. Assim, na NPG, a eficiência recebe uma carga política, democrática e inclusiva que acrescenta a uma suposição de eficiência administrativa.

A última dimensão para a análise da NPG é o accountability. Segundo Campos (1990, p. 37), accountability se caracteriza por representar "[...] a responsabilidade objetiva de uma pessoa ou organização responder perante outras pessoas ou organizações". Em se tratando de accountability na NPG, para Sant'Anna et al. (2019), o Estado é chamado a reconhecer valores e interesses do cidadão, que, por sua vez, avalia políticas públicas exercendo sua capacidade de pressionar o governo a corrigir suas ações. Essa accountability, a qual eles denominaram de multifacetada, deve fazer parte de um acordo formal entre os interessados, em que haja as definições claras de papéis, responsabilidades e mecanismos de execução e responsabilização.

Sinclair (1995) define um conjunto mais amplo de accountability no setor público, que vai além do escopo das dimensões financeiras, incluindo responsabilidade democrática, gerencial, burocrática, profissional e pessoal. Além disso, a autora vê a prestação de contas como múltipla e fragmentada, ou seja, a accountability exige compromissos de outros tipos de prestação de contas. Além disso, sugere que a accountability está sendo continuamente construída.

O surgimento do NPG alterou a coordenação política e administrativa, assim como os tipos de alavancas de controle sobre influência e informação que talvez existissem antes (Christensen \& Lægreid, 2007). Essa maior complexidade dos sistemas de accountability visa remodelar processos mais transparentes (Dickinson \& Glasby, 2010), resultando frequentemente em formas hibridizadas de arranjos de accountability (Bracci, 2014). O trabalho de Skelcher e Smith (2015) começou a explorar essas formas alternativas de hibridismo, empregando o conceito de lógicas institucionais para esclarecer valores institucionais mistos e sustentar que a hibridação nesse contexto emerge de uma pluralidade de racionalidades em jogo nas instituições. 
Em síntese, a NPG permite que a atuação do Estado possa ser permeada pelo objetivo da promoção da qualidade dos relacionamentos e dos processos, o que seria fruto de uma administração pública de enfoque pluralista (aberta para todos). Dessa forma, as redes políticas se configuram num novo modelo situado entre o extremo da regulação (Polanyi, 1980) e da autorregulação do mercado (Smith, 1983), em que as relações estruturais sociais criadas pelo modelo de governança seriam pautadas no consenso e na cooperação, em objetivos projetados em longo prazo, em redes coordenadas e na avaliação dos resultados.

\section{CONCLUSÃO}

Graças à complexidade dos problemas atuais, o Estado está cada vez mais incapaz de resolvê-los sozinho. Suas entranhas burocráticas, sem nenhum demérito ao termo, em muitos casos impede uma articulação mais flexível e ágil, com vista à rápida resolução dos problemas.

Os Estados precisam buscar alternativas junto à sociedade civil, com competências específicas capazes de produzir melhores resultados para a sociedade como um todo. Mas chamar a sociedade civil para o debate público implica uma série de problemas que até então carecem de maior compreensão. Por exemplo, de que forma se pode avaliar a legitimidade dos atores sociais na definição das políticas públicas? Como verificar se as políticas públicas a serem implementadas têm responsividade? Como compreender os processos de democracia deliberativa? Quais são os tipos de lideranças mais importantes para a condução das deliberações políticas? Quais são os principais tipos de conflitos, participação e motivações dos atores? Como se dá a formação das estruturas de governança? Apesar de a NPG fornecer uma proposta com vista a uma sociedade mais cidadã e participativa, as formas de fazer ainda carecem de muitos estudos.

Um ponto a destacar é que não se busca aqui encontrar uma melhor maneira de fazer as coisas. A NPG requer a compreensão do ambiente onde está sendo proposta, os valores culturais da sociedade, as motivações dos atores participantes, as capacidades e as competências desses atores, além de garantir a máxima legitimidade em suas ações. São desafios bastante complexos, mas não se trata aqui de encontrar uma "melhor" NPG. Rejeita-se essa ideia tendo visto a necessidade e o grau de evolução de cada sociedade e Estado, assim como a dificuldade de resolução de cada problema. Dessa forma, até formatos de governança pública numa mesma sociedade e por um mesmo governo podem ser diferentes.

Este trabalho buscou trazer a discussão e a necessidade de pensar e compreender a NPG, além de demonstrar as dimensões para sua melhor compreensão. Tem o intuito, assim, de possibilitar o avanço do tema, destacando que o modelo da NPG não implica a exclusão dos modelos administrativos anteriores. Sua ênfase recai na construção de um modelo mais legítimo e democrático.

Este trabalho abre espaço para diversas lacunas a serem estudadas. Cada dimensão apontada merece ser mais bem estruturada, de forma a permitir ao gestor público possibilidades de execução da NPG. A título de exemplo, a construção do termo accountability no modelo de NPG requer uma abordagem procedimental, amplamente estudada na literatura brasileira (Peixe, Rosa \& Passos, 2018) e bastante difundida da NPM, bem como uma abordagem moral (exclusiva da NPG). Não parece haver dúvidas de que a manutenção e o aprimoramento da democracia passam pela predisposição de uma maior aproximação entre o Estado e a sociedade e, por consequência, pela exigência de um maior grau de accountability por parte do Estado perante a sociedade e na operacionalização do controle das ações governamentais.

Por fim, ressalta-se que os estudos apontam a necessidade de mudar a concepção de governo e o modelo de administração pública aplicada, para um centro de relações e obrigações éticas, pautada no compromisso de fazer um mundo melhor e com sentido. Dito de outro jeito, a literatura vem apontando a emergência de uma forma diferente de governar, e não necessariamente um novo modelo de governo. Isso demanda uma visão ampliada que considere as dimensões não contempladas adequadamente pela literatura tradicional, destacando a importância do governo democrático, do foco no interesse público e do papel de cidadão, objetivando a efetiva coprodução dos valores sociais que devem ser publicamente definidos. Esse é o grande desafio. 


\section{REFERÊNCIAS}

Agranoff, R., \& McGuire, M. (2003). Collaborative Public Management: New strategies for local governments. Washington, DC: Georgetown University Press.

Alford, J., \& Hughes, O. (2008). Public Value Pragmatism as the Next Phase of Public Management. The American Review of Public Administration, 38(2), 130-148.

Amamoto, A. F. S., Pereira, J. R., \& Alcântara, V. C. (2020). Remunicipalização e Coprodução do Bem Público: Perspectivas de Gestão dos Serviços de Água e Saneamento. Cadernos Gestão Pública e Cidadania, 25(81), 1-20.

Bass, B. M. (1985). Leadership and performance. New York, NY: Free Press.

Bingham, L. B., Nabatchi, T., \& O'Leary, R. (2005). The New Governance: Practices and Processes for Stakeholder and Citizen Participation in the Work of Government. Public Administration Review, 65(5), 547-558.

Bohman, J. (1996). Public Deliberation: Pluralism, Complexity and Democracy. Cambridge, MA: MIT Press.

Bozeman, B. (2007). Public Values and Public Interest: Counterbalancing Economic Individualism. Washington, DC: Georgetown University Press.

Bracci, E. (2014). Accountability and governance in social care: The impact of personalisation. Qualitative Research in Accounting \& Management, 11(2), 111-128.

Bryson, J. M., Crosby, B. C., \& Bloomberg, L. (2014). Public Value Governance: Moving Beyond Traditional Public Administration and the New Public Management. Public Administration Review, 68, 445-456.

Campos, A. M. (1990). Accountability: Quando poderemos traduzi-la para o português? Revista da Administração Pública, 24(2), 30-50.

Carneiro, R., \& Menicucci, T. M. G. (2011, dezembro). Gestão pública no século XXI: as reformas pendentes. Textos para Discussão, 1686, 1-76.

Christensen, T., \& Lægreid, P. (2007). The Whole-of-Government Approach to Public Sector Reform. Public Administration Review, 67(6), 1059-1066.

Christensen, T., \& Lægreid, P. (2011). Complexity and Hybrid Public Administration - Theoretical and Empirical Challenges. Public Organization Review, 11, 407-423.

Dagnino, E., Oliveira, A. J., \& Panfichi, A. (2006). Para uma outra leitura da disputa pela construção democrática na América Latina. In E. Dagnino, A. J. Oliveira, \& A. Panfichi (Orgs.), A disputa pela construção democrática na América Latina. São Paulo, SP: Paz e Terra.

Davis, P., \& West, K. (2009). What Do Public Values Mean for Public Action? Putting Public Values in Their Plural Place. American Review of Public Administration, 39(6), 602-618.

Denhardt, R. B., \& Denhardt, J. V. (2003). The New Public Service: serving, not steering. New York, NY: M. E. Sharp.

Dias, T. (2012). Governança Pública: uma concepção teóricoanalítica aplicada no governo do Estado de Santa Catarina a partir da implantação das Secretarias de Desenvolvimento Regional (Tese de Doutorado). Universidade Federal de Santa Catarina, Florianópolis, SC.
Dickinson, H., \& Glasby, J. (2010). Why partnership work doesn't work': pitfalls, problems, and possibilities in English health and social care. Public Management Review, 12, 811-828.

Diefenbach, T. (2009). New public management in public sector organizations: the dark side of managerialistic "en lightenment". Public Administration, 87(4), 892-909.

Dryzek, J. (2010). Foundations and frontiers of deliberative governance. Oxford, UK: Oxford University Press.

Dunston, R., Lee, A., Boud, D., Brodie, P., \& Chiarella, M. (2009). Co-Production and Health System Reform - From Re-Imagining to Re-Making. Australian Journal of Public Administration, 68(1), 39-52.

Faria, C. F. (2000). Democracia deliberativa: Habermas, Cohen e Bohman. Revista Lua Nova, 50, 47-68.

Habermas, J. (1995). Três modelos normativos de democracia. Revista Lua Nova, 36, 39-53.

Hanf, K. I., \& Scharpf, F. W. (1978). Interorganizational Policy Making: Limits to coordination and central control. London, UK: Sage.

Head, B. W., \& Alford, J. (2015). Wicked Problems: Implications for Public Policy and Management. Administration and Society, 47(6), 711-739.

Hernaus, T., Vuksic, V. B., \& Štemberger, M. I. (2016). How to go from strategy to results? Institutionalising BPM governance within organisations. Business Process Management Journal, 22(1), 173-195.

Hufty, M. (2010). Gobernanza en salud pública: hacia un marco analítico. Revista de Salud Pública, 12(1), 39-61.

Jakobsen, M. (2013). Can Government Initiatives Increase Citizen Coproduction? Results of a Randomized Field Experiment. Journal of Public Administration Research and Theory, 23(1), 27-54.

Jessop, B. (1993). Towards a Schumpeterian Workfare State? Preliminary Remarks on post-Fordist Political Economy. Studies in Political Economy, 40, 7-40.

Jørgensen, T. B., \& Bozeman, B. (2007). Public Values: An Inventory. Administration \& Society, 39(3), 354-381.

Kalambokidis, L. (2014). Creating Public Value with Tax and Spending Policies: The View from Public Economics. Public Administration Review, 74(4), 519-526.

Kelly, G., \& Muers, S. (2002). Creating public value. An analytical framework for public service reform. London, UK: Cabinet Office Strategy Unit.

Kissler, L, \&; Heidemann, F. G. (2006). Governança Pública: novo modelo regulatório para as relações entre Estado, mercado e sociedade? Revista de Administração Pública, 40(3), 479-499.

Koliba, C., Meek, J., \& Zia, A. (2010). Governance Networks in Public Administration and Public Policy. Boca Raton, FL: CRC Press/Taylor \& Francis.

Konorti, E. (2012). The 3D transformational leadership model. The Journal of American Business Review, 1(1), 165-175. 
Koppenjan, J. F. M., \& Klijn, E. H. (2004). Managing Uncertainties in Networks. A network approach to problem solving and decision making. London, UK: Routledge.

Lynn, L. E., Jr., \& Malinowska, A. (2018). How are Patterns of Public Governance Changing in the US and the EU? It's Complicated. Journal of Comparative Policy Analysis: Research and Practice, 20(1), 36-55.

Marsh, D., \& Rhodes, R. A. W. (1992). Policy Networks in British Government. Oxford, UK: Clarendon Press.

Moore, M. (1995). Creating public value: Strategic management in government. Cambridge, MA: Harvard University Press.

Nabatchi, T., Sancino, A., \& Sicilia, M. (2017). Varieties of participation in public services: The who, when, and what of coproduction. Public Administration Review, 77(5), 766 -776.

Nabatchi, T., Steen, T., Sicilia, M., \& Brand, D. (2016). Understanding the Diversity of Coproduction: Introduction to the IJPA Special Issue on Coproduction. International Journal of Public Administration, 39(13), 1001-1005.

Osborne, S. P. (2006). The New Public Governance. Public Management Review, 3, 377-387.

Osborne, S. P. (2010). The new public governance: emerging perspectives on the theory and practice of public governance. Abingdon, UK: Routledge.

Osborne, S. P., Radnor, Z., \& Nasi, G. (2013). A New Theory of Public Service Management: Towards a (Public) Service-Dominant Approach. American Review of Public Administration, 43(2), 135-158.

Osborne, S. P., Radnor, Z., \& Strokosch, K. (2016). Co-Production and the Co-Creation of Value in Public Services: A Suitable Case for Treatment? Public Management Review, 18(5), 639-653.

Osborne, S. P., \& Strokosch, K. (2013). It Takes Two to Tango? Understanding the Co-Production of Public Services by Integrating the Services Management and Public Administration Perspectives. British Journal of Management, 24, 31-47.

Ostrom, E. (1972). Metropolitan Reform: Propositions Derived from Two Traditions. Social Science Quarterly, 53(3), 474-493.

Ostrom, E. (1996). Crossing the great divide: coproduction, synergy, and development. World Development, 24(6), 1073-1087.

Paula, A. P. P. (2005). Por uma nova gestão pública: limites e potencialidades da experiência contemporânea. Rio de Janeiro, RJ: Editora FGV.

Peixe, B. C. S., Rosa, C. Filho, \& Passos, G. A. (2018). Governança Pública e Accountability: Uma Análise Bibliométrica das Publicações Científicas Nacionais e Internacionais. Revista Contemporânea de Contabilidade, 15(36), 77-96.

Peters, B. G., \& Pierre, J. (1998). Governance Without Government? Rethinking Public Administration. Journal of Public Administration Research and Theory, 8, 223-224.

Pierre, J., \& Peters, B. G. (2010). Governance, Politics and the State. Houndmills, Basingstoke: MacMillan Press, 2010.

Polanyi, K. (1980). A Grande Transformação: as origens da nossa época. Rio de Janeiro, RJ: Campus.

Provan, K. G., \& Kenis, P. (2008). Modes of network governance: structure, management, and effectiveness, Journal of Public Administration Research and Theory, 18(2), 229-252.
Radnor, Z., Osborne, S., \& Glennonln, R. (1996). Public management theory. In C. Ansell, \& J. Torfing (Eds.), Handbook on theories of governance (pp. 46-60). Cheltenham, UK: Edward Elgar Publishing.

Ramadass, S. D., Sambasivan, M., \& Xavier, J. A. (2018). Collaboration outcomes in a public sector: impact of governance, leadership, interdependence and relational capital. Journal of Management \& Governance, 22(3), 749-771.

Rhodes, R. A. W. (1988). Beyond Westminster and Whitehall. London, UK: Unwin Hyman.

Rhodes, R. A. W. (1996). The new governance: governing without government. Political Studies, XLIV, 652-667.

Rhodes, R. A.W. (1997). Understanding Governance. Buckingham, UK: Open University Press.

Sancino, A. (2016). The Meta Coproduction of Community Outcomes: Towards a Citizens' Capabilities Approach. Voluntas: International Journal of Voluntary and Nonprofit Organizations, 27(1), 409-424.

Sant'Anna, L. T., Alcântara, V. C., Pereira, J. R., Cappelle, M. C. A., \& Tonelli, D. F. (2019). Aproximações entre Governança Colaborativa e Ação Comunicativa: Uma Proposta Analítica de Estudo. Revista de Administração Pública, 53(5), 821-837.

Silva, R. R., Neto, Reis, T. R., \&Ribeiro, A. L. (2020). Perfil da Gestão Municipal no Estado do Pará: Um Olhar a Partir do Índice de Efetividade. Contabilidade, Gestão e Governança, 23(1), 93-106.

Sinclair, A. (1995). The Chameleon of Accountability: Forms and Discourses. Accounting Organisations and Society, 20(2), 219-237.

Skelcher, C., \& Smith, S. R. (2015). Theorizing hybridity. Public Administration, 93(2), 433-448.

Smith, A. (1983). Riqueza das Nações: Investigação Sobre sua Natureza e suas Causas. São Paulo, SP: Abril Cultural.

Sørensen, E., \& Torfing, J. (2007). Theories of Democratic Network Governance. London, UK: Palgrave Macmillan.

Sorrentino, M., De Marco, M., \& Rossignoli, C. (2016). Health care co-production: Co-creation of value in flexible boundary spheres. In: T. Borangiu, M. Dragoicea, \& H. Nóvoa (Eds.), Exploring Services Science: 7th International Conference (pp. 649-659). Bucharest, Romania: Springer.

Sorrentino, M., Sicilia, M., \& Howlett, M. (2018). Understanding co-production as a new public governance tool. Policy and Society, 37(3), 277-293.

Stoker, G. (2003). Public Value Management (PVM): A new resolution of the democracy/efficiency tradeoff. Manchester, UK: Institute for Political and Economic Governance.

Ter Bogt, H. J. (2008). Recent and future management changes in local government: Continuing focus on rationality and efficiency? Financial Accountability \& Management, 24(31), 31-57.

Wallace, M., \& Tomlinson, M. (2010). Contextualizing leader dynamics: How public service leaders endeavour to build influence. Leadership, 6(1), 21-45.

Wiesel, F., \& Modell, S. (2014). From New Public Management to New Public Governance? Hybridization and Implications for Public Sector Consumerism. Financial Accountability and Management, 30(2), 75-205. 
Breno Augusto Diniz Pereira

ORCID: https://orcid.org/0000-0001-7387-5033

Pós-Doutor pelo Centro de Pós-Graduação e Pesquisa em Administração (CEPEAD) da Universidade Federal de Minas Gerais (UFMG); Professor Associado da Universidade Federal de Santa Maria (UFSM). E-mail: brenodpereira@gmail.com

Ivan Beck Ckagnazaroff

ORCID: https://orcid.org/0000-0002-8179-269X

Doutor pelo Doctoral Programme Aston Business School-Aston University; Professor Titular do Departamento de Ciências Administrativas e do Centro de Pós-Graduação e Pesquisas em Administração (CEPEAD) da Universidade Federal de Minas Gerais (UFMG). E-mail: ivanbeck00@gmail.com 